Der Kongress bietet reichlich Gelegenheit zum innereuropäischen Austausch. Sind in Bezug auf die Entwicklung der Hausarztmedizin in Europa Tendenzen festzustellen, aus denen sich Schlüsse für die Schweiz ziehen lassen?

Wonca ist ein Ort der Begegnung, des Austauschs und des Gegenseitig-voneinander-Lernens zur Förderung der Hausarztmedizin in allen Ländern für eine qualitativ hochstehende Gesundheitsversorgung der Bevölkerung. Es gibt in Europa eine starke Tendenz zur gezielten und praxisorientierten Weiterbildung zum Facharzt in Hausarztpraxen anstatt in Spitälern. In Holland und neu auch in Frankreich werden Assistenzärzte mit dem Berufsziel Hausarztmedizin regelmässig $\mathrm{zu}$ hausarztspezifischen Kursen zusammengezogen. Für die Grosspraxen und Notfallzentren, wie sie sich nun auch in der Schweiz entwickeln, dienen uns vor allem Dänemark und Holland als Vorbilder. Die Kon- takte zu diesen Ländern begannen in der Regel an Wonca-Kongressen. Die Vereinigung der Schweizer Jungärztinnen und -ärzte, die ich eben erwähnt habe, ist klar auf Wonca zurückzuführen.

Ist der Kongress ausschliesslich auf Hausärztinnen und Hausärzte ausgerichtet, oder ist das Zielpublikum weiter gefasst? Welche anderen Zielgruppen werden angesprochen, und mit welchen Veranstaltungen?

Das Zielpublikum sind sicher Hausärztinnen und Hausärzte, aber auch ganz besonders Assistenzärztinnen und -ärzte auf dem Weg zur Hausarztmedizin und auch Studierende mit Interesse an Hausarztmedizin. Alle anderen Spezialärztinnen und -ärzte und weitere Berufsgruppen, die sich für ein Gesundheitswesen mit einer starken Hausarztmedizin im Zentrum interessieren, sind sehr herzlich willkommen.

\title{
Röntgenschutzkleidung: Bei «bleifrei» ist Vorsicht geboten
}

\author{
Heinrich Eder ${ }^{a}$, \\ Waldemar Zapf \\ a Dr. Ing., vormals Bayerisches \\ Landesamt für Umwelt, \\ Augsburg \\ b MTR, Kreiskrankenhaus \\ Zwiesel
}

\section{Überblick}

Röntgenschutzkleidung wurde noch vor einigen Jahren überwiegend aus Blei oder Bleiverbindungen hergestellt. Aus Gründen der Umweltproblematik werden zunehmend bleifreie Schürzen entwickelt und in den Handel gebracht. Hierbei spielt auch der Gewichtsfaktor eine Rolle: Bleifreie Schürzen sollen vermeintlich leichter sein als Schürzen aus Blei.

Hinsichtlich der Schutzwirkung wurden bislang alle Schürzen nach dem sogenannten Bleigleichwert (PBGW) beurteilt. Sie sind in 0,25-, 0,35- und 0,50-mmBlei-Äquivalent erhältlich.

Die Frage ist nun, ob das Schutzkriterium «Bleigleichwert» auf bleifreie Schürzen bzw. Blei-Composite-Schürzen (Mischmaterial aus Blei und bleifreien Stoffen) übertragen werden kann.

Die Antwort lautet: Nein! Die Bleigleichwertkennzeichnung einer derzeit benutzten bleifreien Schürze kann deshalb in Bezug auf die tatsächliche Schutzwirkung irreführend sein [1].

Der Bleigleichwert wird nach der internationalen Norm IEC 61331-1 [2,3] im schmalen Strahlenbündel bestimmt. Diese Messmethodik erfasst lediglich die Primärstrahlung und nicht die im Material entstehende Sekundärstrahlung. Blei entwickelt im Energiebereich des diagnostischen Röntgens praktisch keine Sekundärstrahlung, deshalb ist die Methodik dort sachlich richtig. Nicht so bei bleifreien Stoffen:

Zinn, Barium und Antimon - diese Stoffe sind in Bleifrei-Schürzen häufig vertreten - werden bei äusserer Röntgen-Bestrahlung zur Fluoreszenz angeregt. Die Röntgen-Fluoreszenzstrahlung ist wie im sichtbaren Bereich (Beispiel Polarlicht) niederenergetisch, d.h. sie besitzt eine geringere Energie als die primäre Strahlung.

\section{Prudence à propos des vêtements de protection contre les radiations «sans plomb»}

Les vêtements de protection contre les radiations étaient surtout fabriqués, il y a encore quelques années, à partir de plomb ou de composés à base de plomb. On fabrique et on commercialise de plus en plus des tabliers sans plomb pour des raisons de protection de l'environnement. Le facteur poids joue ici aussi un rôle: les tabliers sans plomb seraient prétendument plus légers que ceux avec du plomb. Des mesures montrent toutefois dans beaucoup de cas, que l'exposition aux radiations est plus élevée lors du port de tablier sans plomb que celle des vêtements de protection habituelle qui en contiennent.

Les tabliers sans plomb qui n'ont pas démontré leur effet protecteur selon la norme DIN 6857-1 ne doivent plus être utilisés au cours de procédures de fluoroscopie (interventions, pose de cathéter cardiaque, angiographie, etc.). De nouveaux matériaux, en partie encore en développement, laissent espérer que dans l'avenir ils pourront offrir de facto une protection identique à celle du matériel traditionnel à base de plomb pour un poids plus faible.
Dr. Ing. H. Eder

Am Stadtpark 43

D-81243 München

eder-h@arcor.de 
Konkret bedeutet dies, dass eine Schürze mit einem überwiegenden Anteil an Zinn oder Antimon, die mit 80-kV-Röntgenröhrenspannung bestrahlt wird, bei einer Energie von rd. $26 \mathrm{keV}$ «leuchtet».

Die Fluoreszenz entsteht quasi unmittelbar auf der Haut und hat eine Eindringtiefe von mehreren Zentimetern. Oberflächennahe Organe wie z. B. Haut, Brustdrüsen, Schilddrüse, Testes, aber auch Knochenmark im Bereich des Sternums werden hierdurch zusätzlich belastet [4].

Auch ist die niederenergetische Fluoreszenzstrahlung zumindest im Zellversuch biologisch schädigender als die Primärstrahlung, so dass hierfür ein Strahlungs-Wichtungsfaktor von 1,5 bis 3 eingeführt werden müsste. Dies ist derzeit Gegenstand der Diskussion in den verschiedenen Strahlenschutzgremien $[5,6]$.

\section{Neue Beurteilung für «bleifrei»}

Der oben beschriebene Mangel führte dazu, dass unter der Federführung des deutschen Normenausschuss Radiologie (NAR) eine einheitliche Messvorschrift entstand, die sowohl Bleischutzkleidung wie auch blei-

Abb. 1: Durchlässigkeit (Transmission) von Röntgenschürzen bei 80 kV Röhrenspannung in \% der Dosis auf der Strahlen-Eintrittsseite.

PB: Bleischürze, PBC: Blei-Composite-Schürze, NLG, NLX, NLH: bleifreie Schürzen

Abb. 1a: Messung bei Primärstrahlung nach DIN 6857-1. Gestrichelte Linien: oben Toleranzgrenze nach DIN 6857-1, unten Durchlässigkeit von 0,35 mm Blei.

Abb. 1b: Messung unter Streustrahlung am Alderson-Rando-Phantom unter patientenähnlichen Bedingungen.

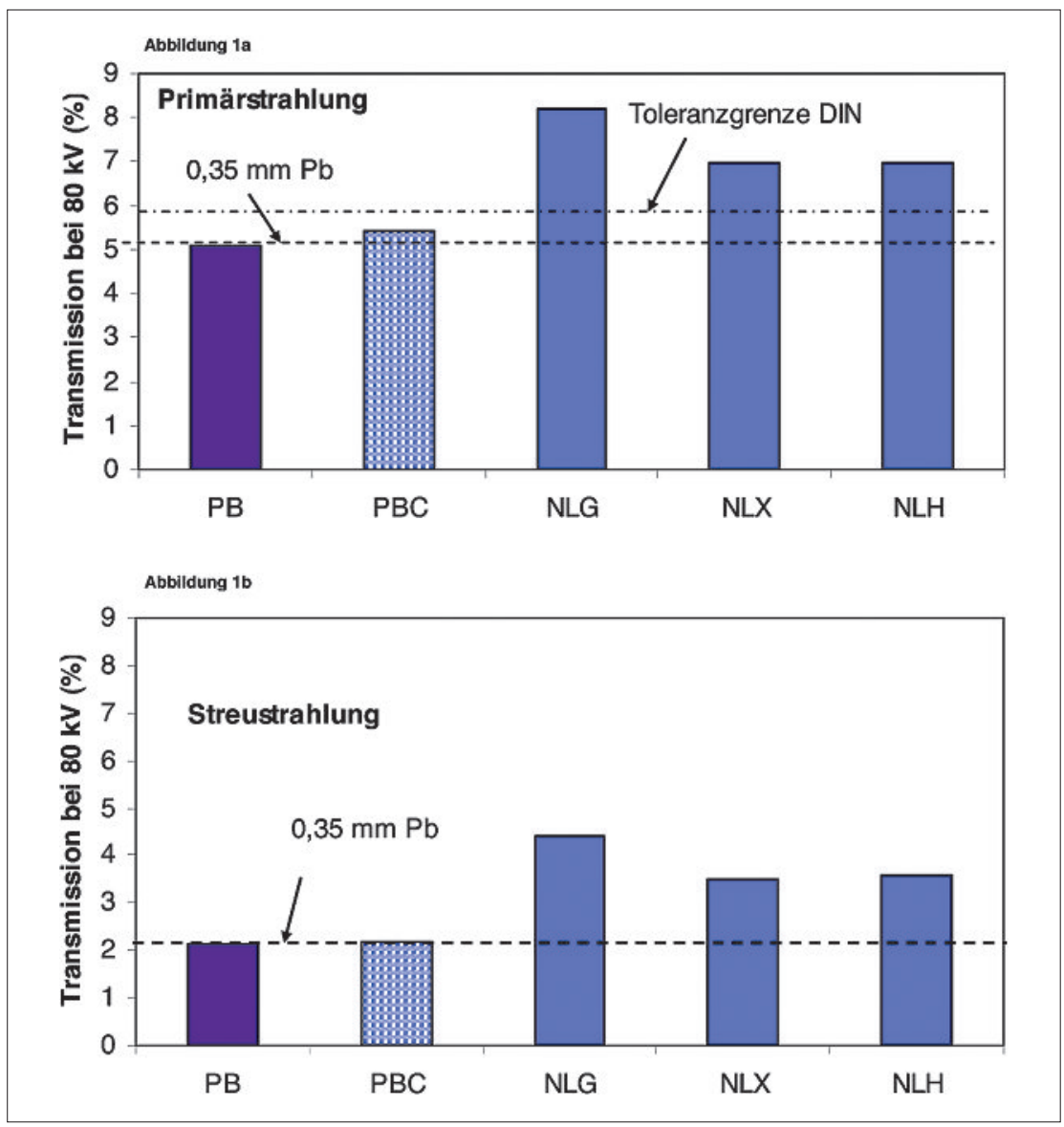

freie oder teilweise bleifreie Schutzkleidung so beurteilt, dass die Materialien hinsichtlich Schutzwirkung vergleichbar werden [7].

Die Norm 6857-1 beschreibt eine Messung im breiten Strahlenbündel, die auch die Sekundärstrahlung erfasst (Anmerkung: genau genommen wird die inverse Geometrie verwendet, die der Messung im breiten Bündel entspricht). Schutzkleidung wird dabei in 4 Klassen eingeteilt: Schutzklasse II wird beispielsweise zugeteilt, wenn die Schutzwirkung im grossen Strahlenfeld in einem Spannungsbereich von 50 bis $120 \mathrm{kV}$ mindestens so gross ist wie diejenige des «Traditionsmaterials» Blei von $0,35 \mathrm{~mm}$ Stärke.

Der Anwender kann also beim Tragen einer nach der neuen Norm geprüften Schürze sicher sein, dass er mindestens den Schutz geniesst, den ihm bisher eine Bleischürze mit entsprechender Dicke geboten hat.

\section{Messungen in der Praxis}

Wir haben handelsübliche Schutzschürzen einer Messung nach der neuen DIN 6857-1 unterzogen.

Es wurden drei bleifreie Schürzen, eine Blei-Composite-Schürze und eine Bleischürze verglichen. In Abb.1a ist die Transmission dargestellt, d.h. der Anteil der primären Strahlung, der hinter der Schürze auf der Haut des Trägers ankommt - einschliesslich Fluoreszenzstrahlung.

Alle Schürzen sind vom Hersteller mit dem Bleigleichwert 0,35 mm Pb gekennzeichnet, so dass der Anwender eigentlich von der gleichen Schutzwirkung ausgehen kann.

Ergebnis: Alle bleifreien Schürzen hielten die Mindestanforderungen nicht ein. Allein die Blei-Composite-Schürze erfüllte die Norm.

Das Bild wird im Vergleich zu Blei noch ungünstiger, wenn man statt der Primärstrahlung Streustrahlung verwendet und damit patienten-äquivalente Verhältnisse schafft. Hierfür wurde ein anthropomorphes Phantom (Alderson-Rando-Phantom) benutzt (Abb. 1b).

Auch unter den Realitätsbedingungen erfüllen die bleifreien Schürzen den Standard nicht. Lediglich die Blei-Composite-Schürze liegt innerhalb der Normbedingung.

Im Mittel kann man - gegenüber Blei oder BleiComposite - von einer anderthalbfachen bis doppelten Belastung ausgehen.

Hier kommt auch wieder das Gewicht ins Spiel: Würde man die de facto leichtere Bleifreischürze auf die gleiche Schutzwirkung bringen, hätte sie etwa wieder das Gewicht einer Bleischürze. Die Physik lässt sich nicht betrügen!

\section{Test mit Personendosimetern}

Die dargestellten Messwerte wurden auch mit amtlichen Personendosimetern - Glas- sowie Filmdosimetern - überprüft. Die Glasdosimeter erfassen die neue Messgröße $H_{p}(10)$. Dazu wurden die Dosimeter jeweils hinter einer Bleischürze und einer Bleifreischürze an- 
gebracht und bei 70 und $100 \mathrm{kV}$ simultan mit Streustrahlung aus dem Phantom belichtet.

Ergebnis: Die Glasdosimeter zeigen hinter der Bleifreischürze gegenüber der Bleischürze eine $60 \%$ höhere Dosis, die Filmdosimeter eine Dosiserhöhung um 90\%.

\section{Zunahme der Organdosen}

Da die Organe für eine Messung kaum zugänglich sind, wurden die Organdosen beim Tragen von Blei- und Bleifreischürzen mit der Monte-Carlo-Methode an den Voxel-Modell-Phantomen Rex und Regina berechnet [4]. Die Erhöhungen gegenüber einer Bleischürze sind in Tabelle 1 angegeben

\section{Eigentest schafft Klarheit}

Wie weiss ein Anwender nun, wie gut seine Schürzen abschirmen? Ein selbst durchgeführter Test kann hier schnell Klarheit schaffen [8]. Dabei geht es nicht um eine Messung nach Norm, sondern einen qualitativen Vergleich zwischen einer Bleischürze und einer bleifreien Schürze. Die meisten Anwender besitzen beides oder können sich eine Bleischürze als Referenzmuster ausleihen (bitte sicherstellen, dass es sich um eine reine Bleischürze handelt!).

Zunächst ist wichtig, dass die zu vergleichenden Schürzen laut Firmenaufdruck den gleichen Bleigleichwert besitzen, also z. B. $0,35 \mathrm{~mm} \mathrm{~Pb}$.

Für die Messung wird ein in der Röntgenabteilung meist vorhandenes eichfähiges Stabdosimeter benötigt. Die Bleischürze bzw. die zu vergleichende bleifreie Schürze wird flach auf dem Röntgentisch ausgebreitet und mit dem Lichtvisier ein Feld von ca. $20 \times 20 \mathrm{~cm}$ eingeblendet. Das Dosimeter liegt im Zentralstrahl unter der Schürze. Es wird eine Belichtung von 75 kV,

\begin{tabular}{lll}
\hline Tabelle 1 & \\
Organ & $\begin{array}{l}\text { Erhöhung der Organdosis } \\
\text { gegenüber einer Bleischürze }\end{array}$ \\
\hline & bei $75 \mathrm{kV}$ & bei $120 \mathrm{kV}$ \\
\hline Mamma & $36,6 \%$ & $44,5 \%$ \\
\hline $\begin{array}{l}\text { Ovarien/Testes } \\
\text { (Mittelwert) }\end{array}$ & $7,2 \%$ & $48,4 \%$ \\
\hline $\begin{array}{l}\text { Schilddrüse } \\
\text { Haut }\end{array}$ & $30,6 \%$ & $39,7 \%$ \\
\hline $\begin{array}{l}\text { Ganzkörper } \\
\text { (effektive Dosis) }\end{array}$ & $45,6 \%$ & $52,5 \%$ \\
\hline
\end{tabular}

rd. 300 mAs empfohlen. Zur Sicherheit sollte die Messung einige Male wiederholt werden

Auf diese Weise kann man die Transmission, also denjenigen Strahlenanteil, der unter der Schürze ankommt, direkt vergleichen. Im Idealfall sollte die Transmission beider Schürzen etwa gleich sein - auf jeden Fall bei Schürzen, die DIN 6857-1 entsprechen.

\section{Fazit}

Die Messungen zeigen die zweifelsfrei höhere Belastung beim Tragen von bleifreien Schürzen.

Bleifrei-Schürzen ohne Nachweis der Schutzwirkung nach DIN 6857-1 sollten bei lang dauernden Durchleuchtungen (Interventionen, Herzkatheter, Angio usw.) nicht mehr verwendet werden. Neuere Materialien, die sich z.T. noch in Entwicklung befinden, lassen hoffen, dass künftig eine dem Traditionsmaterial Blei gleiche Schutzwirkung bei de facto geringerem Gewicht erreicht wird.

\section{Literatur}

1 Eder H, Panzer W, Schöfer H. Ist der Bleigleichwert zur Beurteilung der Schutzwirkung bleifreier Röntgenschutzkleidung geeignet? RöFo - Fortschritte auf dem Gebiet der Röntgenstrahlen und der bildgebenden Verfahren. 2005;177:399-404.

2 IEC 61331-1. Protective devices against diagnostic medical X-radiation - Part 1: Determination of attenuation properties of materials 1994-10.

3 DIN EN 61331. Protective Devices against diagnostic medical X-radiation - Part 1: Dertermination of attenuation properties of materials. 2002.

4 Schlattl, H. Zankl, M. Eder, H. Shielding properties of lead-free protective clothing. Med Physics. 2007;34(11): 4270-80.

5 Schmid E, Regulla D, Kramer H-M, Harder D. The effect of $29 \mathrm{kV} x$ rays on the dose response of chromosome aberrations in human lymphocytes. Radiation Research. 2002; 158:771-7.

6 ICRP-103. Recommendations of the International Commission on Radiological Protection. Oxford: Pergamon Press;2007.

7 DIN 6857-1. Strahlenschutzzubehör bei medizinischer Anwendung von Röntgenstrahlen - Teil 1: Bestimmung der Abschirmeigenschaften von bleifreier und bleihaltiger Schutzkleidung Berlin: Beuth-Verlag (derzeit im Druck).

8 Eder H, Zapf W. Blei-Ersatz oft nicht gleichwertig. Deutsches Ärzteblatt. 2008;105(42):2202-04. 